\title{
Prioritising Tendering Activities for small to medium-sized enterprises (SMEs)
}

\author{
Rahul Swarnkar ${ }^{1}$, Anuj Chaube ${ }^{2}$, Jenny Harding ${ }^{1}$, Bishnu Das ${ }^{1}$, Manoj K Tiwari ${ }^{2}$ \\ ${ }^{I}$ Wolfson School of Mechanical and Manufacturing Engineering, Loughborough University, \\ Loughborough LE11 3TU, UK, \{r.swarnkar,j.a.harding\}@lboro.ac.uk \\ ${ }^{2}$ Department of Industrial Engineering and Management, Indian Institute of Technology Kharagpur, \\ West Bengal, INDIA.
}

\begin{abstract}
The tendering process involves high costs, in terms of time and effort and therefore it is not desirable or sustainable to tender for projects unless the chances of winning are good. Small to medium sized companies do not have enough human resources to enable staff to be dedicated to the job of tending and monitoring market opportunities, and hence company officials have to fit in this job around their usual duties. This paper proposes a knowledge discovery and mining approach to assist the tender offer selection process. Knowledge discovery and mining assures identification and matching of the areas of interest and other criteria of selection of the tender offers, while multi-criteria decision making supports the consideration of other relevant factors for selection.
\end{abstract}

\section{Keywords}

Request for tender selection, tender invitation, small to medium-sized enterprise, SME, analytic hierarchy process, AHP

\section{Introduction}

The development of a comprehensive proposal for a large project can itself be treated as a project. Preparing and writing a comprehensive proposal takes time and is usually expensive. Submission of non winning proposals therefore waste valuable resources and can damage a contractor's reputation, therefore it is not desirable to bid for the contracts for which the chances for winning are low. The above factors make selection of the appropriate tender or proposal to bid upon a very important task.

Large companies may employ groups of people from technical, financial, service-related fields to evaluate tender proposal for feasibility. However small to medium-sized enterprises (SMEs) cannot always afford to spend much of their resources on evaluating tenders. At the same time, in order to maintain their reputation and to remain in business, they can not afford to loose a bid. For a typical SME with only a handful of personnel, certain officials are likely to have to evaluate tenders in addition to their other duties. Based on the observations and feedback from UK based SMEs, the process of tendering involves the following steps:

1. The member of staff responsible for tender offer evaluation receives tender notifications in the form of email/feeds and manually scans them.

2. After a quick assessment, if the decision is made in favour of the preparing a tender, a request is sent to the publisher requesting the pre-qualification questionnaire (PQQ) and other tender documents. (In some cases, the PQQ is attached with the initial offer.)

3. The PQQ is filled and tender bid is prepared for submission.

In the first step, tender notifications are commonly obtained from automated services provided by tender publishing websites. When the staff receive emails with tender information, they manually scan the titles of the tenders, and then for ones which appear appropriate proceed to read the available short descriptions giving consideration to various aspects like application area, the place of performance and the project duration, etc. If they feel that the tender suits the 
company profile and they will be able to achieve the deliverables, they then request further documentation for the tender offer. This process of evaluation is illustrated in Figure 1 with the decision process involved shown by lozenge shaped boxes. In a typical case, the member of staff needs to spend around 1 hour every day in going through this process (assuming 3 minutes is spent in going through each tender description and around 20 offers are received every day), which can be quite difficult for the member of staff to manage around their other responsibilities.

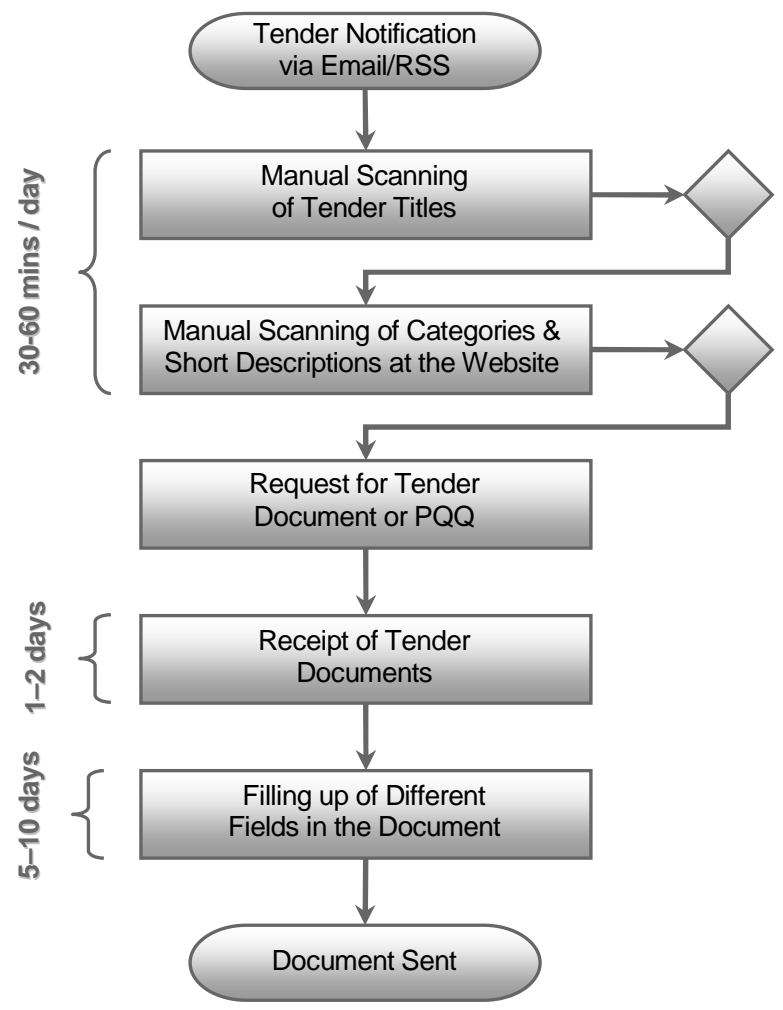

Figure 1: A typical tender evaluation process

The tender notifications are based on the profile information provided by the SMEs subscribed to the tender notification site. It is therefore very important that the subscriber selects an appropriate set of input details. Obviously, an SME would want to get alerts about any tender which is in their area of core expertise. Additionally, they are likely to want to be notified about the tenders which are in areas close to their core expertise. However this increases the number of sectors they can get alerts from and hence escalates the number of alerts received every day. With large quantities of alerts to work through, the above process of evaluation becomes an elimination process rather than a selection process.

A decision on whether to bid for a project by submitting a tender is reached by a process of evaluation, the steps of which include evaluating the title of the tender, then the short description and then the details. The amount of detail and quality of information contained in the email or web feed provided by the automated services depends on what is provided by the originator of the request for tender for them to send. Having a deterministic rule of evaluation has benefits of time saving, however it is easy to be misled by a wrong selection of words for the title and it is impossible to guarantee that all relevant tenders are selected.

A number of different approaches for "tender selection" are found in the literature. However, most of them, if not all, address the problem of supplier/vender selection, i.e. the selection of an appropriate bid out of the incoming ones for a published tender. Vender selection greatly affects the success of an organisation in the market and is one of the most important purchasing 
decisions[Weber, Current, Benton 1991]. Therefore it has been one of the most tackled problems by the operational research community. Weber et al. [Weber, Current, Benton 1991] review literature on this area of work since [Dickson 1966]. The research work in this area over the last 10 years include the use of various methods. Wong et al. [Wong, Holt, Harris 2001] investigate multi-criteria selection approach as opposed to the "lowest price" criteria for the construction industry. Ghodsypour and O'Brien [Ghodsypour, O'Brien 2001] present a mixed integer non-linear programming model to solve the multiple sourcing problem, taking into account the total cost of logistics including net price, storage, transportation and ordering costs. An approach for solving qualitative multi-criteria analysis of with fuzzy pair-wise comparison is presented by Deng [Deng 1999]. Hsieh et al. [Hsieh, Lu, Tzeng 2004] present a fuzzy multicriteria analysis approach for selecting of planning and design alternatives in a public office building. Want et al. [Wang, Chen, Chen 2007] apply incomplete linguistic preference relations to evaluate tender selection criteria and provide decision matrices for making pair-wise comparisons.

Analytical Hierarchy Process (AHP), originally proposed by Saaty in the 1970s [Saaty 1990, Saaty 2008] is one of the most popular methods for addressing multi-criteria analysis/selection problems involving qualitative data and has successfully been applied in real life situations. AHP is a theory of measurement through pair-wise comparisons and relies on the judgement of experts to derive priority scales. Fong and Choi [Fong, Choi 2000] utilise AHP for helping construction clients in identifying contractors with the best potential to deliver satisfactory outcomes. Sha and Che [Sha, Che 2005]develop a partner selection and production-distribution planning model for establishing virtual integration based on AHP, multi-attribute utility theory and integer programming. Kahraman et al. [Kahraman, Cebeci, Ulukan 2003] uses a fuzzy AHP approach to select the best supplier firm for a white good manufacturer against the predetermined criteria using questionnaire and interviews.

All of the research work listed above address the problem of tender selection from the point of view of the contracting authority, e.g local governments or larger companies outsourcing its projects. This research work approaches the tendering problem from the other point of view, i.e. from the supplier's perspective. The problem of "tender offer selection" might look quite different from that of "tender bid selection"; as in this case, the selection process is about picking a suitable job in contrast to picking a suitable candidate to do the job. However, in essence both the problems are multiple criteria decision making problem. Therefore, the knowledge gained from the literature on supplier/vendor selection can be well utilised to address the problem under consideration. This paper proposed a knowledge discovery and mining aided multi-criteria decision making approach to assist the tender offer selection process. The various criteria for selecting/rejecting with appropriate priorities are determined in advance, while the knowledge about the description of job is extracted as the tender appear in market. The next section provides a detailed account of the proposed methodology.

\section{Aiding the Tender Selection Process with AHP}

The process of tendering is highly complex in nature and involves high stakes. Therefore it is not possible (and advisable) to make the entire process automated, however the process of initial scrutiny of tender offers can be computer assisted. The proposed approach for tender offer selection makes use of information extraction from the tender publishing websites using real time text mining and builds its own knowledge base of the tender offers. The knowledge about a company's profile including its core competencies and areas of interests already exists in the database. When a new tender offer appears, it is matched with company's profile and a more targeted notification is sent to the company. The process is illustrated in Figure 2 


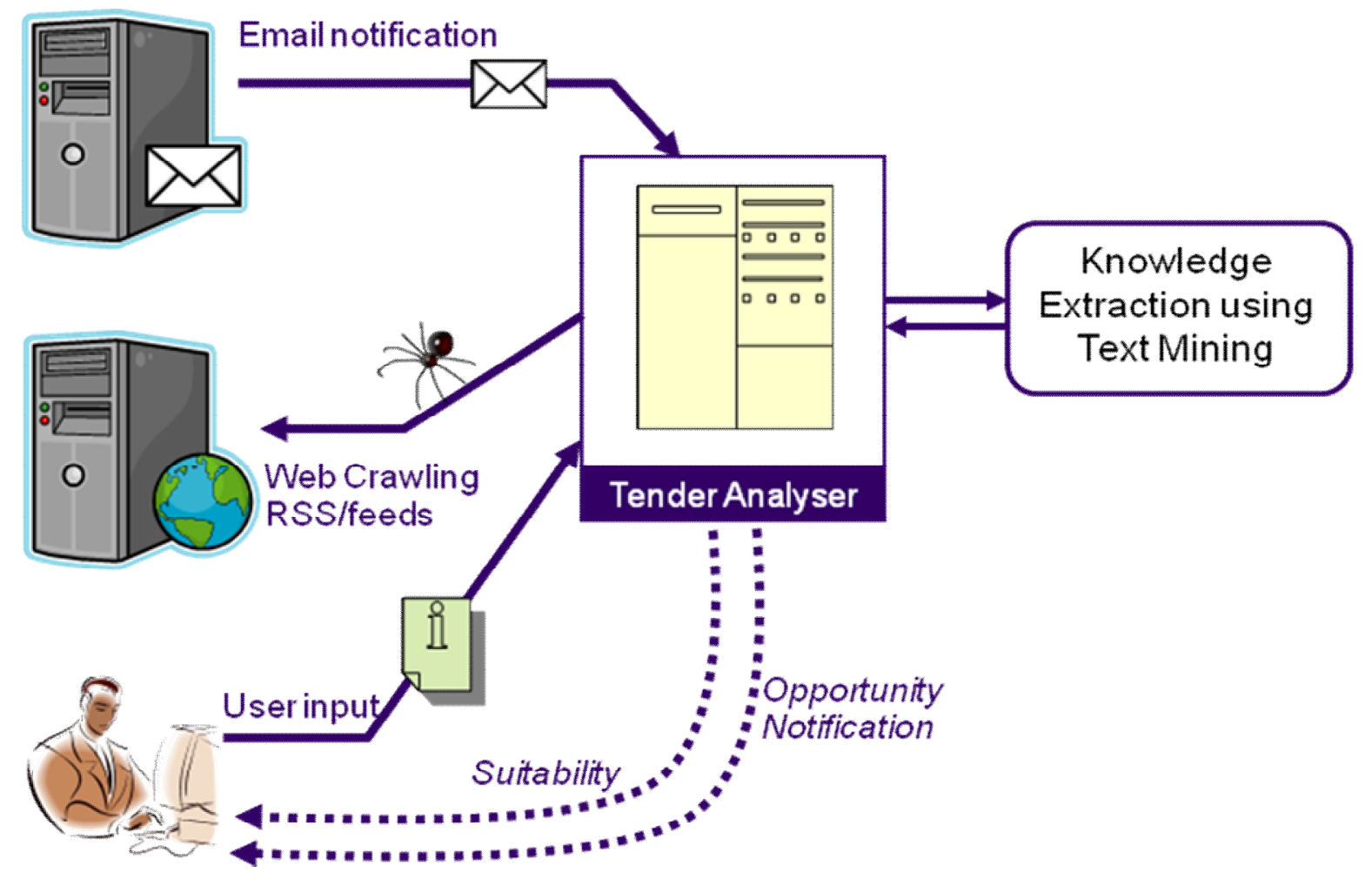

Figure 2: Assisting the tender offer selection

The above mentioned process of "matching" the tender offer to the company's profile is a multicriteria decision process which is based on AHP. In addition to the knowledge about the company's competencies, their preference of selection criteria is learned. For example, a small company may want to submit tenders for projects worth $£ 75,000$ to $£ 1,00,000$ and it may want to operate locally, say within a radius of 40 miles only. As the company does not have enough employees it cannot handle projects a disperse locations. Therefore, it may chose not to bid for a tender for which the place of operation is far away even if the budget falls under its limits. In this case, we observe that the selection criteria of geographical location is preferred over budget of the project.

In a real life case however, we encounter multiple criteria and each one has a preference order. The AHP methodology, which is described as a decision aiding tool for dealing with complex unstructured and multi attribute decision, decomposes a problem into a hierarchy of more easily comprehended sub-problems, each of which can be analyzed independently [Saaty 1990]. Once the hierarchy is built, the decision makers systematically evaluate its various elements, comparing them to one another in pairs. In making the comparisons, the decision makers can use concrete data about the elements, or they can use their judgments about the elements' relative meaning and importance. Fortunately, it is possible for the company officials to tell which of the criteria is preferred over which. In the case of tender offer selection, the main problem is to rate the tender documents according to its suitability. This is shown at the top of the hierarchy in Figure 3. The main problem is divided in multiple small problems, which in this case include rating the tender according to different criteria such as technical, financial, etc. A number of candidate tender offers are compared with each other based on the different criteria. The solution can be represented mathematically as follows. 


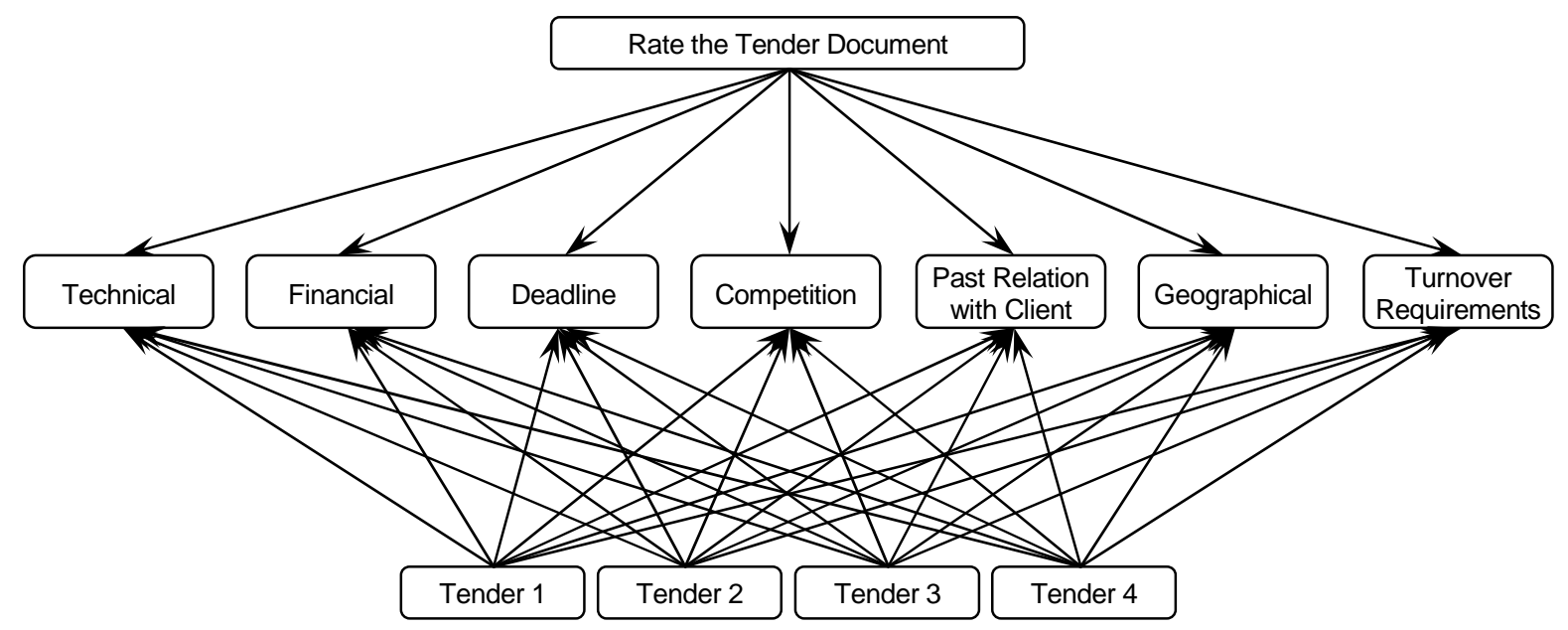

Figure 3: AHP applied on tender offer selection

Let $\quad i \in I$ where $I=\{1, \ldots, T\}$ set of candidate tenders

$j \in J$ where $J=\left\{1, \ldots, P_{i}\right\}$ set of all the properties of tender $i$

$k \in K$ where $K=\{1, \ldots, C\}$ set of criteria set for selecting a tender

Individual properties of the tender correspond to certain criteria for selection and have varied rankings in terms of the suitability to the company. We define these rankings as

$$
R_{i j}=\text { ranking of property } j \text { of tender } i \text { for the company }
$$

and

$$
x_{i j k}=\left\{\begin{array}{l}
1 ; \text { if property } j \text { of tender } i \text { corresponds to criteria } k \\
0 ; \text { otherwise }
\end{array}\right.
$$

If the weight given to different selection criteria is represented as $W_{k}$, the utility of tender $i$ can be calculated as

$$
U_{i}=\frac{\sum_{k \in K} \sum_{j \in J} x_{i j k} R_{i j} W_{k}}{\sum_{k \in K} W_{k}}
$$

The above formulation utilises two sets of information: preferences of selection criteria and preference of options for individual selection criteria. The next section explains the methodology of determining these sets from the vague information provided by the company official.

\section{Understanding the Methodology}

In order to explain the proposed methodology, a set of seven decision criteria are presented: technical area, financial value, completion deadline, competition, past relationship, geographical location and turnover requirement. The preferences of the various options for a certain criteria can be decided by interviewing the company officials and obtaining a rating of each option against other in a matrix form. The eigenvector of this matrix is obtained and normalised to determine the weights of the processes considered [Saaty 1990]. The first $7 x 7$ entries in Table 1 show the input obtained from an example company and the last column shows the overall importance of the criteria.

For each criteria, different options were assigned with different preferences. For example, the SME preferred to work within certain distance from its main site. So the tenders for which the location of performing the job falls within the given distance get a higher priority than others. Table 2 shows example preferences for two selection criteria as high, medium or low. This needs to be quantified and normalised in order to be used for the evaluation. 


\begin{tabular}{|c|c|c|c|c|c|c|c|c|}
\hline Criteria & Technical & Financial & Deadline & Competition & $\begin{array}{c}\text { Past } \\
\text { Relationship }\end{array}$ & Geographical & $\begin{array}{c}\text { Turnover } \\
\text { Requirement }\end{array}$ & $\begin{array}{l}\text { Overall } \\
\text { Rating }\end{array}$ \\
\hline Technical & 1 & 2 & 3 & 4 & 5 & 6 & 7 & 0.3542 \\
\hline Financial & $1 / 2$ & 1 & 2 & 3 & 4 & 5 & 6 & 0.2399 \\
\hline Deadline & $1 / 3$ & $1 / 2$ & 1 & 2 & 3 & 4 & 5 & 0.1586 \\
\hline Competition & $1 / 4$ & $1 / 3$ & $1 / 2$ & 1 & 2 & 3 & 4 & 0.1036 \\
\hline Relationship & $1 / 5$ & $1 / 4$ & $1 / 3$ & $1 / 2$ & 1 & 2 & 3 & 0.0675 \\
\hline Geographical & $1 / 6$ & $1 / 5$ & $1 / 4$ & $1 / 3$ & $1 / 2$ & 1 & 2 & 0.0447 \\
\hline Turnover & $1 / 7$ & $1 / 6$ & $1 / 5$ & $1 / 4$ & $1 / 3$ & $1 / 2$ & 1 & 0.0312 \\
\hline
\end{tabular}

Table 1: Entries in the table show relative preference of criteria and their overall importance

\begin{tabular}{ll|ll}
\hline Technical Area & Preference & Project Value & Preference \\
\hline e-learning; educational services; adult learning & High & $£ 80,000-£ 250,000$ & High \\
web based services; software system; information system & Medium & $£ 20,000-£ 80,000$ & Medium \\
& & $£ 250,000-£ 500,000$ & \\
medical software; management software; programming & Low & $<£ 10,000$ & Low \\
& & $>£ 500,000$ & \\
\hline
\end{tabular}

Table 2: Preferences for options under selection criteria

\section{Application of the Proposed Approach}

The proposed approach for tender evaluation and selection has been applied to create a prototype example. A Java based web crawler fetches data from tender websites and performs text mining to extract useful information like the competencies required, location, completion time etc. and stores in a local MySQL database. The tender analyser module, which has access to both the company preferences information and the local database with tender data performs the analysis to produce the result showing the relevant tenders as shown in Figure 4.

\section{Report: 1225451598379 - Windows Internet Explorer 7}

C: Documents and Settings $\backslash m m r s 3$ My Documents WetBeansProjects $\backslash$ TenderAnalyser $\backslash 123 . h t$

Favorites

Report: 1225451598379

\section{UK-London: computer and related services}

Procurer reference: Not applicable Official EU reference: 18627-2008 Please note: this notice has been published in English only Full Details CONTRACT AWARD NOTICE Services SECTION I: CONTRACTING AUTHORITY I.1) NAME, ADDRESSES AND CONTACT POIN...

https://www.sell2wales.co. uk/notices/displayOJ.html?Noticeld=145818 - $1 \mathrm{k}$ - Cached - Relevance: $51.7 \%$

\section{UK-Abinqdon: repair and maintenance services}

Procurer reference: UVDB-QSN-SERVICES-SEPT-2008 Official EU reference: 240809-2008 Please note: this notice has been published in English only Full Details QUALIFICATION SYSTEM $\square ?$ ? UTILITIES This notice a call for competition: yes SECTION I:...

https://www.sell2wales. $\infty$. uk/notices/displayOJ.html?Noticeld=174601 - 4k - Cached - Relevance: $44.8 \%$

\section{UK-Leeds: computer and related services}

Figure 4: Output from the tender analyser

\section{Concluding Remarks}

The above methodology is being prototyped as a service. The knowledge about selection criteria and criteria preference is acquired from SMEs while the sources of tender text used in our 
prototype implementations are government agencies and tender websites. This approach ensures that a tender notifications are sent to a more targeted recipient while ensuring that all the areas of interest are covered. As the text mining is done on the entire tender text, the search is not misled by the title or the short description.

The initial results obtained from the analysis have been very promising and have been shown to the company officials, who generally agreed with the output. However, some unsuitable tenders have also appeared in the results. The reason for these inaccuracies is thought to be because such tenders were quite verbose and contained many more keywords than needed, resulting in misleading the text mining process. This type of errors however can be omitted with the use of more precise filters at the cost of more coding time and is possible with commercial implementations.

Further research is planned to improve the accuracy of this approach and also determine its potential for raising awareness of business opportunity within networks of collaborating SMEs. This will be carried out as part of the SYNERGY (Supporting highly adaptive Network Enterprise collaboration through semantically-enabled knowledge services) research project which is funded under the EU's $7^{\text {th }}$ Framework Research Programme [SYNERGY 2009].

\section{References}

Deng, H. (1999) "Multicriteria analysis with fuzzy pairwise comparison", Fuzzy Systems Conference Proceedings, 1999. FUZZ-IEEE '99. 1999 IEEE International, pp. 726.

Dickson, G.W. (1966) "An analysis of vendor selection systems and decisions", Journal of Purchasing, vol. 2, no. 1, pp. 5-17.

Fong, P.S. \& Choi, S.K. (2000) "Final contractor selection using the analytical hierarchy process", Construction Management and Economics, vol. 18, pp. 547-557.

Ghodsypour, S.H. \& O'Brien, C. (2001) "The total cost of logistics in supplier selection, under conditions of multiple sourcing, multiple criteria and capacity constraint", International Journal of Production Economics, vol. 73, no. 1, pp. 15-27.

Hsieh, T., Lu, S. \& Tzeng, G. (2004) "Fuzzy MCDM approach for planning and design tenders selection in public office buildings", International Journal of Project Management, vol. 22, no. 7, pp. 573-584.

Kahraman, C., Cebeci, U. \& Ulukan, Z. (2003) "Multi-criteria supplier selection using fuzzy AHP", Logistics Information Management, vol. 16, no. 6, pp. 382.

Saaty, T.L. (2008) "Decision making with the analytic hierarchy process", International Journal of Services Sciences, vol. 1, no. 1, pp. 83-98.

Saaty, T.L. (1990) "How to make a decision: The analytic hierarchy process", European Journal of Operational Research, vol. 48, no. 1, pp. 9-26.

Sha, D.Y. \& Che, Z.H. (2005) "Virtual integration with a multi-criteria partner selection model for the multiechelon manufacturing system", The International Journal of Advanced Manufacturing Technology, vol. 25, no. 7, pp. 793-802.

Synergy - Enabling Collaboration. WWW Page. http://www.synergy-ist.eu/, accessed 01.05.2009.

Wang, T., Chen, L.Y. \& Chen, Y. (2007) "Applying Incomplete Linguistic Preference Relations to Tender Selection", International Conference on Wireless Communications, Networking and Mobile Computing, WiCom 2007, pp. 6734.

Weber, C.A., Current, J.R. \& Benton, W.C. (1991) "Vendor selection criteria and methods", European Journal of Operational Research, vol. 50, no. 1, pp. 2-18.

Wong, C.H., Holt, G.D. \& Harris, P. (2001) "Multi-criteria selection or lowest price? Investigation of UK construction clients' tender evaluation preferences.", Engineering Construction \& Architectural Management (Blackwell Publishing Limited)\}, vol. 8, no. 4, pp. 257. 